\title{
CHARACTERIZATION OF AFFINE RULED SURFACES
}

\author{
by WŁODZIMIERZ JELONEK
}

(Received 15 June, 1995)

0. Introduction. The aim of this paper is to give certain conditions characterizing ruled affine surfaces in terms of the Blaschke structure $(\nabla, h, S)$ induced on a surface $(M, f)$ in $\mathbb{R}^{3}$. The investigation of affine ruled surfaces was started by $\mathrm{W}$. Blaschke in the beginning of our century (see [1]). The description of affine ruled surfaces can be also found in the book [11], [3] and [7]. Ruled extremal surfaces are described in [9]. We show in the present paper that a shape operator $S$ is a Codazzi tensor with respect to the Levi-Civita connection $\hat{\nabla}$ of affine metric $h$ if and only if $(M, f)$ is an affine sphere or a ruled surface. Affine surfaces with $\hat{\nabla} S=0$ are described in [2] (see also [4]). We also show that a surface which is not an affine sphere is ruled iff $\operatorname{im}(S-H I)=\operatorname{ker}(S-H I)$ and $\operatorname{ket}(S-H I) \subset \operatorname{ker} d H$. Finally we prove that an affine surface with indefinite affine metric is a ruled affine sphere if and only if the difference tensor $K$ is a Codazzi tensor with respect to $\nabla$.

1. Preliminaries. For the basic facts concerning affine differential geometry we refer to $[10]$. Let $(\nabla, h, S)$ be the Blaschke structure induced on an affine surface $(M, f)$ in $\mathbb{R}^{3}$ and $\hat{\nabla}$ be the Levi-Civita connection for $h$. Then $\nabla=\hat{\nabla}+K$ where $K$ is a difference tensor. The Blaschke structure induced on a surface $(M, f)$ in $\mathbb{R}^{3}$ is characterized by the conditions

$$
\begin{array}{lc}
\text { (A) } & \operatorname{tr}_{h} K(., .)=0 ; \\
\text { (G) } & R(X, Y) Z=h(Y, Z) S X-h(X, Z) S Y ; \\
\text { (G1) } & R(X, Y) Z=H(h(Y, Z) X-h(X, Z) Y)+\hat{\nabla} K(X, Y, Z)-\hat{\nabla} K(Y, X, Z) ; \\
\text { (C1) } & \nabla h(X, Y, Z)=\nabla h(Y, X, Z)=-2 h(K(X, Y), Z) ; \\
\text { (C2) } & \hat{\nabla} S(X, Y)-\hat{\nabla} S(Y, X)=K(S X, Y)-K(X, S Y) ; \\
\text { (R) } & h(S X, Y)=h(Y, S X) ; \\
\text { (K) } & {\left[K_{X}, K_{Y}\right] Z=-J(h(Y, Z) X-h(X, Z) Y),}
\end{array}
$$

where by $J=\frac{1}{2} h(K, K)$ we denote the Fubini-Pick invariant. The number $H=\frac{1}{2} \operatorname{tr} S$ is called an affine mean curvature of the affine surface $(M, f)$ with induced structure $(\nabla, h, S)$.

2. Surfaces in $\mathbb{R}^{3}$ with symmetric $\hat{\nabla} S$. We begin with the following lemma.

Lemma 1. Let $(\nabla, h, S)$ be an induced Blaschke structure with symmetric $\hat{\nabla} S$. Then $\left[K_{X}, S\right]=K_{X} \circ S-S \circ K_{X}=0$ for every $X \in T M$ and $S=H I$ or $J=0$, which means that $(M, f)$ is (locally) an affine sphere or a ruled surface.

Proof. From the Codazzi equation (C2) we get

$$
K(S X, Y)=K(X, S Y)
$$

Glasgow Math. J. 39 (1997) 17-20. 
Hence $S K(X, Y)=K(S X, Y)$ and $\hat{\nabla} S=\nabla S$ (which follows from $[4$, p. 181]). Consequently

$$
\left[K_{X}, S\right]=0
$$

for every $X \in T M$. Equation (2) implies

$$
S \circ\left[K_{X}, K_{Y}\right]=\left[K_{X}, K_{Y}\right] \circ S
$$

for every $X, Y \in T M$. Using (K) we obtain

$$
-J S(h(Y, Z) X-h(X, Z) Y)=-J(h(S Y, Z) X-h(S X, Z) Y) \text {. }
$$

Hence $J=0$ or

$$
h(Y, Z) S X-h(X, Z) S Y=h(S Y, Z) X-h(S X, Z) Y .
$$

Taking the trace of (5) with respect to $Y$ we get $h(S X-(\operatorname{tr} S) X, Z)=-h(S X, Z)$ which means $S=H I$. Let us recall that an affine surface with $J=0$ is a quadratic or a ruled surface (see [11], [7]). This completes the proof.

Our next lemma characterizes ruled surfaces that are not spheres. that

LEMma 2. Let $(\nabla, h, S)$ be the induced Blaschke structure on a surface $(M, f)$ such

$$
\operatorname{ker}(S-H I)=\operatorname{im}(S-H I) \subset \operatorname{ker} d H
$$

Then $(M, f)$ is ruled.

Proof. From $\left({ }^{*}\right)$ it follows that there exists a local frame such that (see [12]):

$$
\begin{gathered}
S E_{1}=H E_{1}, \quad S E_{2}=H E_{2}+E_{1} ; \\
h\left(E_{i}, E_{i}\right)=0, h\left(E_{1}, E_{2}\right)=\varepsilon, \varepsilon \in\{-1,1\} .
\end{gathered}
$$

By (7) it is clear that $\hat{\nabla}_{X} E_{i} \| E_{i}$. Hence, (6) and $\left(^{*}\right)$ imply:

$$
\begin{aligned}
\hat{\nabla} S\left(E_{2}, E_{1}\right)= & \left(E_{2} H\right) E_{1}-(S-H I)\left(\hat{\nabla}_{E_{2}} E_{1}\right)=\left(E_{2} H\right) E_{1} \\
\hat{\nabla} S\left(E_{1}, E_{2}\right) & =\left(E_{1} H\right) E_{2}-(S-H I)\left(\hat{\nabla}_{E_{1}} E_{2}\right)+\hat{\nabla}_{E_{1}} E_{1} \\
& =-(S-H I)\left(\hat{\nabla}_{E_{1}} E_{2}\right)+\hat{\nabla}_{E_{1}} E_{1} .
\end{aligned}
$$

From the Codazzi equation (C2) we get

$$
K\left(E_{1}, S E_{2}\right)-K\left(S E_{1}, E_{2}\right)=K\left(E_{1}, E_{1}\right) \| E_{1}
$$

and $K\left(E_{1}, E_{1}\right)=\beta E_{1}$. By $(7)$ and $(\mathrm{A})$ we have $K\left(E_{1}, E_{2}\right)=0$ and consequently $0=$ $\nabla h\left(E_{1}, E_{1}, E_{2}\right)=-2 \beta \varepsilon$. Hence $\beta=0$. It follows that $K_{E_{1}}=0$ and in view of $(\mathrm{K}), J=0$ which means that $(M, f)$ is ruled since, from $(*), S \neq H I$.

In Lemma 3 we show that a ruled surface which is not a sphere satisfies condition $(*)$.

Lemma 3. Let $(M, f)$ be a surface with $J=0$ and $S \neq H I$. Then $(M, f)$ is ruled, condition $\left(^{*}\right)$ is satisfied, and $S$ is a Codazzi tensor with respect to $\hat{\nabla}$. 
Proof. Since $J=0$ we have a local frame (see [5]) such that

$$
h\left(E_{i}, E_{i}\right)=0, h\left(E_{1}, E_{2}\right)=1 \text {, }
$$

and

$$
K_{E_{1}}=0, K\left(E_{2}, E_{2}\right)=\gamma E_{1},
$$

where $\gamma$ is a smooth function and consequently, in view of (G1), we also have

$$
\begin{gathered}
R\left(E_{1}, E_{2}\right) E_{1}=S E_{1}=H E_{1} ; \\
-R\left(E_{1}, E_{2}\right) E_{2}=S E_{2}=H E_{2}+\left(3 \omega\left(E_{1}\right)-E_{1} \gamma\right) E_{1},
\end{gathered}
$$

where $\hat{\nabla}_{X} E_{1}=\omega(X) E_{1}$. From (12), (13), (14) it follows that $\left[S, K_{X}\right]=0$ for every $X \in T M$. Hence $\nabla S$ is symmetric, $E_{1} H=0$ and condition $\left(^{*}\right)$ holds.

We have now proved:

THEOREM. For an affine surface $(M, f)$ with the induced Blaschke structure $(\nabla, h, S)$ the following conditions are equivalent:
(a) $\hat{\nabla} S$ is a symmetric tensor;
(b) $\nabla S=\hat{\nabla} S$;
(c) $\left[K_{X}, S\right]=0$ for every $X \in T M$;
(d) $S=H I$ or $J=0$;
(e) $\operatorname{im}(S-H I)=\operatorname{ker}(S-H I) \subset \operatorname{ker} d H$ or $S=H I$;
(f) $(M, f)$ is ruled or is an affine sphere.

Proof. These are straightforward consequences of the above lemmas.

REMARK 1 . It is easy to show that an affine surface $(M, f)$ with induced structure $(\nabla, h, S)$ is projectively flat if and only if

$$
\hat{\nabla} S(X, Y)-\hat{\nabla} S(Y, X)=(X H) Y-(Y H) X
$$

Hence as an application of our result we get:

COROLlaRY 1. The only projectively flat affine surfaces with constant affine mean curvature are affine spheres and ruled surfaces with constant curvature. A ruled surface is projectively flat if and only if its affine mean curvature is constant.

I obtain the last part of this Corollary by different methods in [6].

We finish by characterizing affine spheres with vanishing Fubini-Pick invariant.

Proposition. Let $(M, f)$ be an affine surface with induced Blaschke structure. Then $(M, f)$ is a sphere with vanishing Fubini-Pick invariant if and only if $\nabla K$ is a symmetric tensor.

Proof. As $\nabla=\hat{\nabla}+K$, we obtain

$$
\hat{\nabla} K(X, Y, Z)-\hat{\nabla} K(Y, X, Z)=\nabla K(X, Y, Z)-\nabla K(Y, X, Z)-2\left[K_{X}, K_{Y}\right] Z .
$$

As $\left[K_{X}, K_{Y}\right] Z=-J(h(Y, Z) X-h(X, Z) Y)$, from $(\mathrm{G} 1)$ we get

$$
R(X, Y) Z=\nabla K(X, Y, Z)-\nabla K(Y, X, Z)+(H+2 J)(h(Y, Z) X-h(X, Z) Y) .
$$


If $\nabla K$ is symmetric then (16) yields (on using formula $S X=\operatorname{tr}_{h} R(X,$.$) .)$

$$
S X=(H+2 J) X .
$$

From (17) it follows $J=0$ and $S=H I d$. Now if $(M, f)$ is an affine sphere with $J=0$ then from (15) and (G1) it is clear that $\nabla K$ is a symmetric tensor.

COROLLARY 2. An affine surface with an indefinite induced Blaschke metric is a ruled affine sphere if and only if $K$ is a Codazzi tensor relative to the induced Blaschke connection $\nabla$.

REMARK 2. Let us note that $\nabla K=0$ if and only if $\hat{\nabla} K=0$ and $J=0$; hence an affine surface $(M, f)$ with $\nabla K=0$ is a Cayley surface or a quadric (see [8]).

\section{REFERENCES}

1. W. Blaschke, Vorlesungen über Differentialgeometrie II (Springer, Berlin, 1923).

2. F. Dillen, Locally symmetric complex affine hypersurfaces, J. Geometry 33 (1988), 27-38.

3. F. Dillen, A. Martinez, F. Milan, F. G. Santos and L. Vrancken, On the Pick invariant, the affine mean curvature and the Gauss curvature of affine surfaces Results in Math. 20 (1991), $622-642$.

4. W. Jelonek, Affine surfaces with parallel shape operators, Ann. Polon. Math. LVI.2. (1992), 179-186.

5. W. Jelonek. The local structure of affine spheres in $\mathbb{R}^{3}$, Preprint 505, Polish Academy of Sciences (1992).

6. W. Jelonek, Projectively flat connections and rigidity of affine spheres in Selected problems of Mathematics, Cracow University of Technology, Vol. 6 (1995), 161-169. Math.)

7. W. Jelonek, Ruled surfaces in affine differential geometry (to appear in Demonstratio

8. M. Magid and K. Nomizu, On affine surfaces whose cubic forms are parallel relative to the affine metric, Proc. Japan Acad. 65, Ser. A (1989), 215-218.

9. A Martinez and F. Millan, On affine maximal ruled surfaces, Math. Z. 208 (1991), 635-644. $165-178$.

10. K. Nomizu and U. Pinkall, On the Geometry of Affine Immersions, Math. Z. 195 (1987),

11. P. A. Schirokov and A. P. Schirokov, Affine Differentialgeometrie (Teubner, Leipzig, 1962). $177-194$.

12. L. Vrancken, Affine surfaces with constant affine curvatures, Geom. Dedicata $\mathbf{3 3}(1990)$,

INSTITUTE OF MATHEMATICS

TeChNical University of Cracow

WARSZAWSKA 24

31-155 Krakow, Poland 